\title{
Epistemic and poietic intentional processes
}

\author{
Józef Lubacz ${ }^{1}$
}

Received: 29 April 2020 / Accepted: 20 January 2021 / Published online: 4 February 2021

(c) The Author(s) 2021

\begin{abstract}
We examine the intentional processes that correspond to conceptualizations of activities performed by subjects with the intention of achieving an objective. Taking as its basis a general framework of intentional processes, two types of such process are considered: epistemic ones, aimed at acquiring knowledge about something, and poietic ones, aimed at bringing about something. The "something" is understood as anything that the processes can pertain to: a physical, mental or abstract object, a phenomenon, a state of affairs, etc. The generic features of such processes are discussed, with focus on: (1) features that are common for epistemic and poietic intentional processes as well as on features that differentiate them, (2) the dynamic and static features of the processes, and on (3) issues involved in controlling the progression of the processes towards intended objectives. The latter issue is the essential part of our considerations, the two former establish the necessary conceptual framework. The presented analysis aims at shedding light on these aspects of human intentional activities which can be considered virtually independent of any specific area of human intentional activity, be it natural sciences, humanities or technology.
\end{abstract}

Keywords Intention $\cdot$ Process $\cdot$ Epistemic processes $\cdot$ Poietic processes

\section{Introduction}

We shall be considering a certain type of activity on the part of individual human beings - the sort performed with an intention-with a determination to reach some objective. The activities will be conceptualized as intentional processes-processes that are undertaken in an attempt to realize subjects intentions. In accordance with the title of this paper, two types of intentional processes are considered: epistemic and poietic intentional processes. The terms "epistemic" and "poietic" are derived from Ancient Greek, and are treated here as possessing a quite general context of

Józef Lubacz

j1@ tele.pw.edu.pl; jozef.lubacz@ @w.edu.p

1 Faculty of Administration and Social Sciences, International Center for Formal Ontology,

Warsaw University of Technology, Plac Politechniki 1, 00-661 Warsaw, Poland 
applicability and meaning-especially the term poiesis (from the Greek $\pi$ ore $\tilde{\imath} \nu$ to make), which was originally applied principally to arts and crafts, although not without some relation to notions of knowledge and know-how. The relation between "knowledge that" and "knowledge how" in Ancient Greek thought is considered, e.g. by Hintikka (1974).

Epistemic and poietic processes are, respectively, assumed to undertake realization of epistemic and poietic intentions. An epistemic intention is directed towards the objective of acquiring knowledge about something, whereas a poietic intention is directed towards bringing something about. That "something" may be an object, a phenomenon or state of affairs of the world external to the intending subject, or it may pertain to the subject itself. Of course, knowledge acquired by a subject about something affects the subject, so an epistemic process itself has some poietic effect. But conversely, realizing a poietic intention requires knowledge about what is to be achieved and how, so epistemic and poietic processes are in fact interdependent. Thus, obviously, real-world intentional epistemic and poietic activity involves interdependent superposition of component epistemic and poietic sub-processes. Considering the latter as idealized, "pure" epistemic and poietic processes is helpful in interpreting the interdependence and differentiating features of real-world activities. The introduced idealized conceptualization reveals the essential features of intentional epistemic and poietic activities considered as phenomena which progress in time (rather than as timeless acts or events). This, in turn, involves an analysis of the generic principles of controlling the progression of epistemic and poietic processes towards their intended goals. The analysis, presented in Sect. 4, is the most important result of our considerations. The preceding sections constitute a conceptual framework for the considerations from Sect. 4. The framework is based on multiple ontological and epistemic notions that are involved in the introduced conceptualization of intentional processes and their epistemic and poietic instances. It is also important to consider that a poietic sub-process usually requires some pre-existing "know that", and that an epistemic sub-process usually requires some pre-existing "know how". This is, however, a different aspect of the considered distinction and interrelation of poietic and epistemic processes because the pre-existing knowledge may be assumed to be acquired in some separate, preceding processes.

Our approach to analyzing epistemic and poietic activity as unfolding processes necessitated introducing some specific concepts that are related to the interpretation of the terms "process" and "intention". We thus begin with some interpretative clarifications of how these terms are understood in our investigation.

There is no agreed upon understanding of the term process. This is hardly surprising, given that the word appears in such diverse areas as philosophy, the natural sciences, mathematics, engineering, the social sciences and the humanities. The general, common understanding of the term is that it refers to any phenomenon of change occurring in time, without any reference to what might be causally implicated within this as regards its conditions of possibility, with the consequence that such an understanding, though relatively all-encompassing, also seems quite superficial. The notion of process has acquired a certain popularity fairly recently, yet the underlying issue has a long tradition associated with it-one already recognizable in Ancient Greek (and also Eastern) thought, insofar as these were already 
concerned with the problem now sometimes referred to as the relationship of static and dynamic features of being and beings. Applied to processes, the essence of this relationship may be spelt out as being the relation between their changeability, i.e. their inherent dynamism, and some "mechanism" of change that can be considered a static constituent of this or that process. For example, if one considers the observed changeability of nature, its static constituent may be identified with the mechanisms of evolution, or with divine intervention, or be interpreted as resulting from some deterministic or randomized pattern, etc. At the same time, the interpretation of the interplay of dynamic and static features of processes will, of course, depend on the particular phenomena being conceptualized as processes. The relation of the dynamic and static constituents of processes involves fundamental, still controversial, metaphysical questions, cf. Stout (2016) and Steward (2013); an overview of discussion pertaining to the involved questions is contained in Stout (2018). For reasons indicated in the following section such metaphysical analysis does not have to be involved in the proposed conceptualization of intentional processes.

In our considerations we interpret intentions as a complex, multi-aspect phenomenon, in the sense considered by Hyman (2015): human action and agency involves a complex interplay of psychological, physical, intellectual and ethical components. According to our interpretation, analogously to the interpretation of Bratman (2009), intention is an attitude which guides the conduct of actions and provides their consistency in realizing the actions' objectives. Hyman and Bratman do not, however, consider the processual aspects of human activities, so our interpretation is appropriately adapted to express time-related progression of processes. This issue is considered in Sect. 2. Considering the adopted interpretation of intention, we shall not embark here upon an analysis of the complex issue of the relation between the notions of "intention" and "intentionality", cf. Jacob (2019) and Setiya (2018), and in particular on Brentano's and Husserl's considerations that are focused on the phenomenon of consciousness.

Although we focus on features of intentional activity of individuals, it is assumed that if an individual activity is performed as part of some collective undertaking aimed at fulfilling a common objective, then any individual taking part in that enterprise will be assumed to have been allocated the task of achieving a sub-objective that is itself a component of the shared undertaking. We assume also that if an individual undertakes the task allocated, then that task is performed intentionally. Thus, in the following we shall not distinguish between cases where the process corresponding to a subject's intentional activity is undertaken individually, and cases where it arises as a consequence of participation in some collective project.

The analysis of epistemic and poietic intentional processes from the perspective adopted here is virtually not considered in the works mentioned above nor in the context of "process philosophy", cf. Seibt (2018). The traditional focus of epistemology is on the sources, limits and results of knowledge, rather than on the processes that lead to its acquisition, i.e. from the perspective of epistemic intentional processes realized by individuals that are considered in the present paper. Obviously, this perspective differs from the viewpoint on processes of acquiring and growth of knowledge represented e.g. the by the Kuhn's analysis of actual practices and "revolutions" in science, Popper's methodological considerations, Lakatos' scientific 
programs or by a more recent approach based on "conceptual spaces" proposed by Gärdenfors and Zenker (2013). A comprehensive reflection on analytic and critical approaches to social aspects of scientific knowledge has been presented by Niiniluoto (2018).

In general, poietic activity, although was always present in human societies, has attracted much lesser ontological and methodological reflection than epistemic activity. On the other hand, there has been a considerable focus on issues associated with the impact of the development of technology on society, economy and civilization generally, cf. Scharf and Dusek (2003). Given the contemporary development of knowledge, especially scientific knowledge, as well as its practical application in many areas, the mutual dependence of epistemic and poietic processes upon on one another is quite obvious, especially the dependence of science and technology, cf. Morawski (2019). In this context two related papers of Staples $(2014,2015)$ are important. Staples has presented a comprehensive analysis of the essential ontological and methodological issues involved in engineering, contrasted with problems involved in science. In our own considerations scientific and engineering activities are regarded as interrelated epistemic and poietic processes. Although Staples focuses on engineering and science, his understanding of these areas of creative activity is quite general and analogous to our own understanding of epistemic and poietic activity: science tries to understand the world, whereas engineering tries to change it. Staples focuses on engineering knowledge, theories and methods in dealing with artefacts and contrasts them with the role of conceptual frameworks in science. In particular he convincingly highlights the fact that engineering has its own kind of knowledge and theories which are similar but essentially different to the conceptual frameworks in science in terms of their role, structure and validity criteria. Our considerations will also refer to these issues, although from a conceptual perspective that exposes the features of epistemic and poietic processes that are virtually independent of any specific area of application, with special focus on the relation between the dynamics and statics of processes and on controlling their progression towards intended objectives. The presented analysis aims at shedding light on these aspects of epistemic and poietic intentional activities which can be considered virtually independent of the particular objectives and objects that are considered in so diverse areas such as natural sciences, humanities and technology.

In the following we introduce the proposed conceptual framework in a top-down stepwise manner, beginning in the next section with a conceptualization of intentional processes which, together with additional assumptions, constitutes the basis for the analysis of epistemic and poietic processes presented in Sect. 3 and Sect. 4. In Sect. 3 we introduce the generic constitutive components of epistemic and poietic processes which are basically common for the two process types; the introduced concepts are illustrated with simple examples. Section 4 seeks to outline the essential distinguishing features of epistemic and poietic processes-principally as these relate to the controlling of how such processes unfold or progress in the direction of fulfilling their intended objectives; the involved issues are illustrated with an appropriate extension of the examples introduced in Sect. 3. The paper then concludes with a concise summary of the introduced conceptualization of epistemic and poietic intentional processes and presents some remarks concerning the possible impact 
of the considerations presented in fostering a better understanding of the essential features of human intentional activity in diverse areas.

\section{Intentional processes}

An intentional process is undertaken in an attempt to realize a subject's intentionwith a determination to reach some objective. In the following, if not confusing, we use shorter expressions: "a process realizes an intention" and "realization of an intention". As explained in Sect. 3, the realization of an intention usually involves concurrent mental and physical activity, although the realization of a process entirely internal to the mental sphere may be also considered. An intention need not be realized, but if it is to be realized it must be executable in the sense that it enables the realization of a process directed at achieving the intention's objective (although the process might not achieve the objective if, for example, the intention is dropped or the objective turns out to be unrealizable for some reason or other). Such intentions will be referred to as executable intentions. The following considerations, then, specifically concern processes understood to be realizations of executable intentions.

To be executable, an intention must be directed towards an objective that specifies not only what is to be achieved, but also how it is to be achieved-at least to the extent necessary to enable initiation of the intention's execution. Thus, if not misleading, the terms realizing an executable intention, an intention and an intended objective will be used interchangeably. Where real-life intentional processes are concerned, and especially if they are genuinely creative ones, the initial specification of the "what is to be achieved" and the "how it is to be achieved"" may be quite vague, becoming more precise or even being altered as a consequence of events internal or external to consciousness. Thus, an executable intention constitutes, so to say, a guideline according to which a process is to be animated with a view to realizing the relevant objective. That guideline may exhibit various degrees of specificity, but even if precisely determined (e.g. in the form of a plan or pattern) it may be modified, or even significantly changed or aborted, as the process in question unfolds. The intention of a process may thus evolve as the process progresses, but it is assumed to be stable until it has in fact changed. The realization of the processi.e. its dynamics - therefore may result from the actualization of an intention that counts as stable in this sense. Put another way, the dynamic features of a process depend on its static constituent, which may itself undergo changes as the unfolding of the process progresses. In effect, then, the statics and dynamics of the intentional processes under consideration here are interdependent. The interplay between a process's intention and its realization carries with it a seemingly paradoxical consequence: the current state of a process may depend not only on past states, which is quite usual and thus not surprising, but also on future developments as determined by the relevant intention. In other words, the "present" may depend on the "past" and on the "intended future". This, however, is an inherent feature of the intentional processes under examination here.

One of the principal questions associated with attempts to conceptualize processes concerns what counts as decisive when considering a process as one entity 
rather than a sequence of distinct activities or sub-processes. This, of course, is an example of the classical problem of identity. There may be different answers to this, depending on the type of phenomena the conceptualization is thought to explain or model. For example, in Whitehead's process metaphysics, cf. Whitehead (1929), the identity problem is tackled with the aid of atemporal objects and the interference of a god in the "occasions of experience" which underlie the temporal features of processes. In this conceptualization, we are dealing with an immortal but non-eternal god, who is in some respects a temporal and in some respects an atemporal entity: one that effectively directs the processes shaping the world and imparts identity to those processes.

Another approach, rather less known or influential, was presented by Ingarden (2013). In his ontology, apart from processes, two other types of temporally determined entities are distinguished: events and "objects persisting in time". The latter are considered to be "... existentially the most potent, since in abiding they can overcome the lapse of time and can serve as existential support for the remaining two types of temporal objects". In his comprehensive analysis, Ingarden concludes that the existence of such objects is indispensable if processes are to preserve their identity in spite of their inherent changeability in time. The criterion of identity of a process is thus identified with a certain constitutive component of it that is responsible for ensuring the stability of certain of its features even while these are interrelated with others subject to change. In other words, the identity of the process follows from the interrelation of its dynamic features with its static features. This is in line with the conceptualization adopted above.

As has been mentioned in the introduction, we do not go here into detailed metaphysical issues that are discussed e.g. in Stout (2018) because in the present context the identity of an intentional process can be established with reference to the changeable intention lying behind the process itself. The evolution of the intention may be interpreted as a sequence of intention-states, each enduring for a distinguishable time-interval. The question, now, is what the criterion of identity of such a sequence of intention-states will be. Two answers may be considered: either (1) the intention-states are instances of a common "meta-intention" or (2) some type of morphism between the successive intention-states is to be assumed. The latter seems quite natural-and, in fact, indispensable-if, for example, a process is initiated with a vaguely specified intention that then gains in detail as the process itself unfolds. A controlled transformation of the intention will be required, especially if the increase in detail requires altering or augmenting the forms of expression of the intention. This must therefore involve morphisms that are assumed to maintain the identity of the intention behind it even as the process itself evolves. The former scenario, on the other hand, might well appear simpler, but in fact this is not obviously the case, because a "meta-intention" would have to be general enough to be independent of any kind of particularization. From the point of view of the many genuinely creative and innovative real-world processes involved in contemporary scientific and engineering activity, this is quite unrealistic. Hence case (1) cannot be assumed independent of case (2). 


\section{Generic constituents of epistemic and poietic processes}

In this section we introduce additional components to the conceptualization of intentional processes presented in the previous section. This constitutes the basic generic conceptual framework for both epistemic and poietic processes. We illustrate the framework with simple examples: a sculptor's poietic activity and an epistemic activity of somebody who tries to figure out whether an observed stick dipped in water is really bent.

It is assumed that the intention of a process is realized with component processive activities. These are mental and physical activities that act on appropriate substances-acting, respectively, on a mental or physical substance composed (in broadly Aristotelian terms) of the appropriate form and matter. In principle, both activity types will be active throughout the unfolding of the process, and usually concurrently so, although a processual unfolding entirely internal to mental activity could also be contemplated if, for example, our reflection on something has yet to be expressed or communicated, or has not yet been so in its entirety.

Of course, knowledge is not exclusively acquired through effecting some specific intention, and not all changes effected by humans are intentional. The processes considered here are of a kind that will not pertain directly to cases of non-conscious or non-intentional epistemic and poietic activity. Such activity may, however, take place simultaneously with intentional activity and influence the latter. Similarly, non-act-based forms of consciousness, such as mood or attitude, may exert an influence on intentional acts of consciousness. Thus the unfolding development of real-world epistemic and poietic processes is a complex interplay of various factors with diverse impacts-ones not consciously directed towards achieving an intended objective. Factors of this sort are not taken into explicit consideration, but can be assumed to be aggregated within the consciously directed unfolding of a process in the direction of an intended objective (that itself may be evolving, too).

As an illustration of the introduced conceptual framework let us consider the poietic activity of a sculptor on the one hand, and the epistemic activity of somebody looking at a stick partly immersed in water on the other. We shall begin with the former case (which seems to be a favorite-though superficial-example used to illustrate Aristotle's "four causes").

Suppose that a sculptor begins work with some provisional, only vaguely determined intention. In the course of the activity, the intended end may become more precisely determined, or be completely altered or even dropped altogether. Now consider the first of these scenarios. The activity begins with some conceptual working out, which is then followed by acting on some physical material. Assuming that the sculptor's mind is not a tabula rasa, the conceptualization will depend on experience, working habits, certain artistic styles and fashions, etc. All these are components of the mental substance underpinning the process associated with the intention. Acting on some physical material involves being reliant upon the sculptor's skills, working habits, stylistic preferences, etc.-so as the process unfolds, there will be a relation of two-way influence between the sculptor's mental and physical activities. 
In the second case, someone sees a stick dipped in water as bent, and wants to know whether the stick is actually bent or only seems so due to an illusory optical effect. Such a cognitive question is a component of an intention that is itself based on prior experience and knowledge. The question is easily resolved by pulling the stick out of the water. Whatever the result, the epistemic activity involves an interaction of two process-related substances and activities: one mental and one physical.

In both of these examples, interdependent mental and physical activities are involved, so with respect to this feature the epistemic and poietic processes being considered cannot be said to differ. Obviously, the mental activity in question is active throughout the entire process of realization of the epistemic and poietic activities in question, with the exception of any "breaks"-periods of suspended activity-or periods when the process is realized "automatically", e.g. through some technical means. Such cases may, however, be passed over here, as they do not introduce any new or additional points of interest.

\section{Controlling the unfolding progression of epistemic and poietic processes}

In the considerations presented so far, no essential distinction between epistemic and poietic processes has been invoked. Such a distinction only really becomes apparent when one examines certain specific features of the realization of these processes leading to the achievement of an intended objective. The examined features are illustrated with adequately extended examples from the previous section.

An epistemic intention is directed towards the objective of acquiring knowledge about something whereas a poietic intention is directed towards bringing something about. The progression of the processes results in some realization of the appropriate intentions - in some realized outcome, which is further referred to as the realized intention. The intentions may evolve as the processes progress, and so may the realization of the intentions. The progression of the processes involves an evaluative confrontation of what was intended with what is actually realized, i.e. a confrontation of the intention with how it is realized.

The realization of an epistemic intention is the knowledge acquired with some mental and/or physical perception means pertaining to the something that the intention was directed to. We will refer to the knowledge so acquired as the realized epistemic intention. As the process progresses the intention is confronted with the realized intention. The realization of a poietic intention is the something that is brought about according to the intention and the perception of that thing with some mental and/or physical means. We will refer to the brought about thing and the perception of it as the realized poietic intention. As the process progresses the intention is confronted with the perception of the thing that was brought about. The perception of the thing brought about in a poietic process may be interpreted analogously as the knowledge pertaining to something that is acquired in an epistemic process.

To illustrate these general considerations, in what follows we use the aforementioned two examples. 
As the sculptor's activity progresses, the intermediately achieved results-i.e. whatever may have been intermediately realized-will be evaluated with respect to the intended ones on the basis of how the results are perceived by the sculptor. In other words, some realized intention will be evaluated with respect to the (current) intention. If the sculptor is not satisfied with the evaluation, the realization of the intention may be modified and then reevaluated. An unsatisfactory evaluation may also lead to modification of the intention. Whatever happens, it is the evaluation of the realized intention with respect to the intention that can trigger a modification of the sculpture or the intention, or both. The crucial point is that the realized intention is evaluated with respect to the intention, not the other way around.

Now consider a modified "stick in water" example. As before, the intention of the observer is to decide whether the stick is really bent, but now it is assumed that the stick cannot be pulled out of the water. The observer views the stick from different angles and, in effect, sees the stick as bent differently, or even as straight. In other words, the perception of the stick depends on the angle from which it is observed. Thus, the realized intention may depend on how the stick comes to be perceived as a consequence of the physical activity of observing the stick from different angles. The observer evaluates the intention with respect to the realized intention. If the evaluation is inconclusive, further observation may be undertaken. Consider also another possible situation: the observer concludes that his or her prior knowledge about the considered effects is too crude a basis for making a decision, and that a modification of the "background knowledge" informing the original intention is therefore now called for. Whatever the situation, the unfolding progression of the process depends on the evaluation of the intention with respect to the realized intention-not the latter with respect to the former, as was the case in the sculpture example.

Note that even in these simple illustrative examples the interrelation of epistemic and poietic tasks is quite evident. The observer of the stick must plan and perform certain physical experiments before the evaluation in question can be performed; this is a poietic task. The sculptor, before the evaluation in question can be performed, must have already acquired some knowledge about how to interpret the results of the physical realization of the intention; this is an epistemic task. This kind of interrelation obtains in most real-word activity in sciences, humanities and engineering. Consider the following examples.

If a physicist intends to acquire knowledge about some phenomenon based on some experimental enquiry he or she has to plan and execute an experiment; this is a poietic task. The epistemic intention usually involves some prior knowledge and hypothesis concerning the considered phenomenon. The intention is evaluated with respect to the result of the experiment, i.e. with respect to the realized intention. The progression of the epistemic process depends on the effect of the evaluation. Essentially the same type of relations obtain in the case of an intention to gain knowledge about the performance of some implemented legal regulations, e.g. legal regulations concerning car traffic. This requires planning what and how is going to be observed and then execution of the plan; this is a poietic activity. The regulations are implemented with some assumed objectives and expected performance; these constitute a prior knowledge and hypothesis of the epistemic intention. The result of the observation of the performance of the implemented legal system constitutes the realized 
intention with respect to which the intended performance is evaluated. Depending on the evaluation result, the epistemic enquiry may be terminated or continued with a modified observation plan or may lead to a conclusion that the regulations should be modified. In the latter case, the modification, if undertaken and implemented, is a poietic task that is essentially analogous to engineering tasks which involve a design and implementation of what was designed, which in turn involves evaluation whether the implementation conforms to the design; this is an epistemic task. Consider finally, that although epistemic enquiry in the humanities differs in many respects from that of the sciences and engineering, it is still a progression of an epistemic process which is controlled in effect of some evaluation of what is intended versus what was realized. The evaluation involves a confrontation, for example, of the researcher's hypothesis about something with available documented evidence and with the work of other investigators.

Although the illustrative examples and interpretations cannot be considered as typical or paradigmatic they indicate that the processes associated with epistemic and poietic forms of activity differ as to the "direction" of evaluation of the intention and realized intention: in epistemic processes the intention is evaluated with respect to the realized intention, whereas in poietic processes the realized intention is evaluated with respect to the intention. We shall therefore hereon refer to these distinct relations obtaining between the intentions and realized intentions as, respectively, the epistemic relation and the poietic relation. These relations are the essential features responsible for conceptual differentiating the two types of intentional process from one another. Note that this is analogous to the contrast introduced by Searle (1983) with respect to beliefs and desires, where beliefs are characterized by a "mind-to-world direction of fit", and desires by a "world-to-mind direction of fit". Searle's characterization in fact pertains to certain specific components of the mental activities involved in epistemic and poietic processes.

It is also worth noting that the famous "shopping list" considered by Anscombe (1957) may serve as an intuitive illustration of epistemic and poietic relations. According to the terminology we have proposed, a shopping list is a specification of an poietic intention of shopping. The perceived effect of shopping, i.e. the realized intention, is evaluated with respect to the shopping list: i.e. with respect to the intention. The shopping list may also be considered an element of the epistemic intention of someone engaged in observing the shopper and preparing a list of what has been bought. In this case, it is the original shopping list that is evaluated with respect to the list of what has actually been purchased: i.e. the intention is evaluated with respect to the realized intention.

In the case of complex real-world epistemic and poietic processes, apart from the relevant mental and physical activities, a presentational activity will also be involved. The latter is assumed to be associated with a presentational substance consisting of the appropriate material and form, as in the case of the mental and physical activities previously considered here. The presentational activity and substance are natural components of poietic and epistemic processes that require some plan or design to serve as, so to speak, an "interface" between the mental and physical activities involved. A presentational substance, expressed with a traditional blueprint or some computerized means and form, constitutes a storage and communication 
medium: this may prove indispensable where group-work is involved, and/or where formalized (e.g. mathematical) means are used to express and realize the intention behind the process.

As far as the unfolding progression of real-world epistemic and poietic processes is concerned, the results of mental activity will be transformed into input data for the presentational activity, and the results of the latter transformed in turn into input data for the mental activity. Thus, a bi-directional feed-forward and feed-back relation obtains. In general, such interactions also occur between presentational and physical activities, and between the latter and mental activities. In consequence, it would be unrealistic to assume that we are simply dealing with a sequential succession of mental, presentational and physical activities. It is also important to note that these activities should not be equated with phases of the realization of processes of the kind used to describe pragmatically oriented methodologies for performing epistemic and poietic activities, cf. Wynn and Clarkson (2017).

Expressing the intention and the realized intention may require some specific, more or less sophisticated language and formal structure, these usually being dependent on the area of application of the epistemic and poietic processes in question. Obviously, such things will differ for, say, the engineering tasks associated with designing a bridge or a computer-not to mention what is involved in acquiring knowledge about, for instance, patterns of biological evolution or economic systems. The substance of the presentational activity may thus vary in essential ways between differing applications of poietic and epistemic processes, making it virtually impossible to arrive at any general conclusions in this regard. It is nevertheless possible to formulate some general principles governing the interaction of the presentational activity with the mental and physical activities involved-ones that are of fundamental importance when it comes to controlling the unfolding progression of epistemic and poietic processes.

Controlling the unfolding progression of such processes requires that two basic kinds of guiding principles (rules, mechanisms, controlling points, etc.) are established: principles governing the transformation of results as these pass between component process activities of different sorts (i.e. mental, presentational and physical), and principles for evaluating the desired quality-related features of such transformations. Obviously, the mental, presentational and physical activities "speak different languages", so establishing formalized transformation principles (such as would result in, say, an isomorphism or homomorphism) between such ontologically differing activities (and their associated substances) is rarely feasible, cf. Lubacz (2019). Hence, we must resort to the use of heuristic rather than formal relations. On top of these difficulties, there is also that which is associated with the ontological diversity typically encountered as internal to this or that individual type of activitybased process. Consider, for example, the poietic intention of constructing a chair. The intention should take into account various aspects and requirements, such as the chair's function, construction materials, durability and aesthetic style. All of these require essentially different means of conceptualization and description, which must nevertheless eventually be integrated so as to form a consistent design. Even if a strictly formalized mode of conceptualization and description is feasible with respect to some particular requirements and aspects, a formalized integration will 
still prove to be unrealistic —even in such a seemingly simple instance as the design and production of a chair. In practice, then, heuristic methodological approaches, based on a mixture of formalization and experience, offer the only viable solution.

Some basically analogous difficulties bear on epistemic intention, though these are usually more selective in the sense that they only pertain to selected phenomena and not to any internally consistent totality of multiple phenomena. This reflects the fact that it can be hard to decide what such a totality should consist of, or how internal consistency can be ensured with respect to the phenomena constituting the totality in question. For example, a unified theory of macro-scale and micro-scale physical phenomena (gravity and quantum phenomena) remains unavailable, and it is far from clear which specific phenomena should be considered components of an internally consistent totality.

The problems just outlined will obviously impact on the evaluation of the consistency of the transformation of results as we pass between the component activities of epistemic and poietic processes-and they will, in consequence, also affect the evaluation of the epistemic and poietic relations involved. If some formalization of the transformations is feasible, then strict mathematical methods can be applied to provide consistency. It is, however, unrealistic to expect that real-world epistemic and poietic processes could ever be fully controlled using strict, formalized means. Such means, even if indispensable, are usually only applicable to selected aspects of activity-based processes and their mutual relations.

The ultimate objective as regards controlling the progression of epistemic and poietic processes will be the evaluation of the epistemic and poietic relations themselves: i.e. the relations of appropriateness obtaining between the intention and the realized intention in all phases of the unfolding of a given process. Such an evaluation will usually be performed via some testing procedure, which will examine whether some predefined criteria of conformity between the two entities are met. Such a testing procedure is usually referred to as verification. In many real-life cases, it may result not only in the answers "yes" or "no", but also "can't decide". In the case of epistemic processes, these answers are naturally associated with questions of the form "Is it true that...?", i.e. with truth conditions. In this respect, poietic processes do not differ essentially from epistemic processes, so it is somewhat surprising that the notion of truth rarely appears in reflections on the former. It is sometimes even claimed that truth-related criteria are inapplicable-e.g. where engineering activity is concerned-and that only usefulness criteria are relevant. Staples $(2014,2015)$ discusses this issue and emphasizes the importance of truth conditions in engineering.

Such verification may be carried out for any intermediate effect pertaining to the realization of an intention, with the results of this intermediate verification constituting one of the drivers for the unfolding progression of the process in question. Moreover, apart from verification, epistemic and poietic processes may also involve validation: i.e. checking whether the results of a process obtained some features that might be virtually impossible to foresee and/or specify within the intention. Here it should be noted that the role of validation is quite frequently not distinguished sufficiently clearly from that of verification. According to a popular intuitive formulation, verification checks "whether a thing has been done right", while validation 
checks "whether the right thing has been done". Validation, treated at a sufficiently general level, also links up epistemic and poietic processes with ethical questions.

As has already been mentioned, mental, presentational and physical activities should not be confused with phases of the execution of a process. The latter are certainly in fairly common use: e.g., in conceptualizations of product-related engineering processes. The so called "life-cycle" of products is split into consecutive phases, such as product requirements, specification, design, implementation, exploitation and disposal. These life-cycle phases are usually assumed to be mutually dependent, thanks to feed-forward and feed-back relations, and are also associated with a variety of engineering methodologies and means of realization. Although numerous sophisticated variants of life-cycles for engineering processes have been proposed, cf. Kroes (2002), Wynn and Clarkson (2017), Wierzbicki and Nakamori (2007), these rarely turn out to be realistic with respect to real-world circumstances - especially in the case of genuinely creative, innovative forms of engineering. Life-cycle models do nevertheless prove helpful when it comes to managing complex engineering processes, especially when these require group-work. The description of a product's life-cycle usually focuses on methodological aspects and procedures specific to particular phases, rather than the issues involved in feed-forward and feed-back relations obtaining between such phases. This is, in fact, the most difficult aspect of things, because such phases usually "speak different languages" and are associated with divergent methodological traditions, frameworks and tools. In light of the conceptualization introduced in this paper, such difficulties can be interpreted as being directly entailed by the fact that the phases in question involve different component processive activities and substances. Whatever the particular interpretation appropriate for various fields of application - say, bridge construction, or the implementation of legal systems - may be, the essence of the difficulty will be the same: it is the challenge of establishing adequate principles and criteria for governing interactions occurring between quite diverse processive activities.

\section{Concluding discussion}

We conclude our considerations with a concise summary of the introduced conceptualization of epistemic and poietic intentional processes and then show how the conceptualization may be used to interpret some of ontological and methodological issues considered by Staples $(2014,2015)$ with respect to engineering, i.e. a particular poietic intentional process. Next, we present general remarks concerning possible directions of extending our work with the aim of fostering a better understanding of human intentional activity in diverse areas.

The essential features of the processes considered here are ones that follow from the fact that such processes are assumed to be driven by executable intentions such as specify both an intended objective (the "what") and the means to achieve it (the "how"). The intention of a process may be altered as the process progresses. Until such an alteration occurs, however, the intention is assumed to constitute a static principle, in accordance with which component processive activities are performed. In effect, then, the dynamics of the process is guided 
by a static intention, but this is one which is at the same time itself subject to dynamic behavior. Thus, on our proposed conceptualization, the dynamics and statics of intentional processes are interrelated, and this interrelation itself confers an identity upon the process in question in spite of its inherent changeability.

Component processive activities (be they mental, presentational or physical) are performed on associated substances, where these will possess the appropriate form and material. This, together with the interdependence of the statics and dynamics of processes, establishes a significant connection between "process philosophy", construed as focusing on why and how things come to be as they are, and "substance metaphysics", whose principal concern is with why things simply are as they are.

These general observations are intended to pertain to intentional processes insofar as these furnish the subject matter of the conceptual framework needed to make sense of epistemic and poietic processes. Apart from their having different objectives, what essentially differentiates such processes is their being associated with features intrinsic to either epistemic or poietic relations obtaining between the intention and the realized intention that are involved in the processes. The assessment of such relations is performed as the process unfolds, with the results of the evaluation constituting the principal driver of its unfolding progression. With epistemic processes, the intention is evaluated with respect to the realized intention, whereas with poietic processes the realized intention is evaluated with respect to the intention. All the same, in spite of this fundamental dissimilarity, epistemic and poietic processes remain mutually dependent: a realization of epistemic intentions will involve poietic tasks, while a realization of poietic intentions will involve epistemic tasks.

The considerations presented by Staples (2014) may be interpreted within the conceptual framework introduced here. Staples introduces a model of engineering knowledge which consists of engineering theories mapped on the Popper's "three worlds" ontological framework. The engineering theories are means that are used by mental, presentational and material activities in controlling the progression of a poietic (engineering) process towards its intended objective, i.e. are used in verification procedures that evaluate the poietic relation (the realized intention vs. the intention) as the process progresses. The engineering theories are used to reason about the mutual relations between the following entities: $(a)$ Usage Situations, $(b)$ Understood/Intended Requirements, $(c)$ Requirement Specification, (d) Design Specification, $(e)$ Understanding of Performance" and $(f)$ Artefact as Built. The entities $c$ and $d$ may be considered as components of the poietic intention that are expressed with the presentational substance, whereas the entities $e$ and $f$ are components of the realized intention: $f$ is what is realized (physical substance) and $e$ expresses how it is perceived (mental/presentational substance). On the one hand the entity $a$ is a component of the realized intention if it is considered in the context of $f$ (of what is realized), but on the other hand it may be considered as a component of the poietic intention if this context is somehow expressed within $c$ and/or $d$. Analogous options pertain to the entity $b$ : if it is interpreted as a component of $c$ and/or $d$ then it belongs to the poietic intention (mental/presentational substance), whereas if it is interpreted as how the requirements are perceived then it is a component $e$ of the realized intention (mental/presentational substance). These options are not explicitly 
considered in Staples (2014) but become quite evident in the analysis presented in Staples (2015).

Staples (2015) introduces a taxonomy of falsification (in the Popperian sense) in engineering. In the conceptual framework of poietic processes a falsification obtains if the realized intention does not conform to the intention. In the Staples's taxonomy, in accordance with the above $a-f$ entities, the lack of conformance may pertain to: Usage Situation vs. Artefact as Built, Requirements Specification vs. Usage Situation, Design Specification vs. Artefact as Built, Requirements Specification vs. Design Specification, Usage Situation vs. Understood Requirements, Understood Requirements vs. Requirements Specification, Design Specification vs. Understanding of Performance, Understanding of Performance vs. Artefact as Built. Stables emphasizes the fact that in engineering there is a wider range of responses to falsification than in science and there are many more appropriate potential corrective reactions to falsification. This follows from the fact that engineering theories deal with various sorts of requirements, which are not part of the ontology of scientific knowledge. This observation pertains to virtually any real-world poietic process.

The conceptualization of epistemic and poietic processes set out above certainly seems straightforwardly applicable to engineering and the sciences, but could also be applied to other areas of human creative activity. Epistemic processes in physics, medicine and the humanities, for example, obviously differ in many respects, especially in terms of their specific intentions, component processive activities, and criteria for evaluating the results of the activities they involve-as do the poietic processes involved in, for instance, the implementation of legal frameworks, economic systems and technological artefacts. Nevertheless, the most important and general conclusions entailed by the considerations elaborated here seem pretty much independent of which specific areas of application one happens to be dealing with. At the same time, though, it would certainly be worth seeking to uncover specific commonalities, diversities and interrelation with regard to epistemic and poietic processes in particular areas of application, as well as in activities involving interdisciplinary, cross-disciplinary or meta-disciplinary problems.

Understanding general features of epistemic and poietic processes, and especially those associated with the relationship between their static components on the one hand and their dynamic behavior on the other, represents an epistemic challenge in its own right. When it comes to evaluating how realistic our conceptual modelling is, above and beyond theoretical considerations of the sort outlined above, a degree of observation of real-word processes is also bound to prove indispensable. The theoretical model, if realistic enough with respect to at least some chosen aspect of real-world processes, could be used to perform computerized simulations or emulations that might, in turn, themselves provide insights not achievable by purely theoretical means. Acquiring such knowledge, at least in such a degree of understanding that has been reached with respect to the features and interrelation of technology and science, would require researchers and practitioners with various forms of expertise to be involved, and the planning of the relevant research activities to be conducted in both top-down and bottom-up terms. This, of course, is a highly ambitious task, but if undertaken may foster hopes for a better understanding of human intentional activity, enabling us to avert possible negative outcomes like those so convincingly 
set out several decades ago by Snow (1961) and characterized, famously, as the phenomenon of "two cultures". Certainly, it is plausible to think that the number of "cultures" has increased since Snow first presented his own thoughts, and that the negative consequences of this increasing fragmentation have deepened and broadened, in which case any reflections leading to a better understanding of general, "culture-independent" features of epistemic and poietic processes would surely appear worthwhile.

Acknowledgements I would like to thank my colleagues from the International Center for Formal Ontology (Warsaw University of Technology, Faculty of Administration and Social Sciences) for their encouragement and advice. Special thanks to Zbigniew Król for inspiring discussions and suggestions. I also thank the anonymous reviewers whose careful comments greatly improved the content and the readability of this text.

Open Access This article is licensed under a Creative Commons Attribution 4.0 International License, which permits use, sharing, adaptation, distribution and reproduction in any medium or format, as long as you give appropriate credit to the original author(s) and the source, provide a link to the Creative Commons licence, and indicate if changes were made. The images or other third party material in this article are included in the article's Creative Commons licence, unless indicated otherwise in a credit line to the material. If material is not included in the article's Creative Commons licence and your intended use is not permitted by statutory regulation or exceeds the permitted use, you will need to obtain permission directly from the copyright holder. To view a copy of this licence, visit http://creativecommons.org/licen ses/by/4.0\%.

\section{References}

Anscombe, G. E. M. (1957). Intention. Cambridge, US: Harvard University Press.

Bratman, M. (2009). Faces of Intention. Cambridge: Cambridge University Press.

Gärdenfors, P., \& Zenker, F. (2013). Theory change as dimensional change: conceptual spaces applied to the dynamics of empirical theories. Synthese, 190(6), 1039-1058.

Hintikka, J. (1974). Knowledge and the Known. Berlin: Springer.

Hyman, J. (2015). Action, Knowledge, and Will. UK: Oxford University Press.

Ingarden, R. (2013). The Controversy over the Existence of the World, vol. I, translated and annotated by Arthur Szylewicz, Peter Lang

Jacob, P. (2019). Intentionality. In E. N. Zalta (Ed.), The Stanford Encyclopedia of Philosophy (Winter 2019 edn.). https://plato.stanford.edu/archives/win2019/entries/intentionality/.

Kroes, P. (2002). Design Methodology and the Nature of Technical Artefacts. Design Studies, 23(3), $287-302$.

Lubacz J. (2019). The Application of Category Theory to Epistemic and Poietic Processes. Category Theory in Physics, Mathematics, and Philosophy, M. Kuś, B. Skowron (eds.), Springer Proceedings in Physics

Morawski, R. Z. (2019). Technoscientific Research: Methodological and Ethical Aspects. De Gruyter.

Niiniluoto, I. (2018). Social aspects of scientific knowledge. Synthese, 197(1), 447-468.

Scharf, R. C., \& Dusek, V. (2003). Philosophy of Technology. Blackwell Publishing.

Seibt, J. (2018). Process philosophy. In E. N. Zalta (Ed.), The Stanford Encyclopedia of Philosophy (Summer 2019 edn.). https://plato.stanford.edu/archives/sum2020/entries/process-philosophy/.

Searle, J. R. (1983). Intentionality. Cambridge, US: Cambridge University Press.

Setiya, K. (2018). Intention. In E. N. Zalta (Ed.), The Stanford Encyclopedia of Philosophy (Fall 2018 edn.). https://plato.stanford.edu/archives/fall2018/entries/intention/.

Snow, C. P. (1961). The Two Cultures and the Scientific Revolution. Cambridge University Press.

Staples, M. (2014). Critical rationalism and engineering: ontology. Synthese, 191(10), 2255-2279.

Staples, M. (2015). Critical rationalism and engineering: methodology. Synthese, 192(1), 337-362.

Steward, H. (2013). Processes, Continuants, and Individuals. Mind, 122, 781-812. 
Stout, R. (2016). The Category of Occurrent Continuants. Mind, 125, 41-62.

Stout, R. (2018). Processes, Experiences, and Actions. Oxford University Press.

Whitehead, A. N. (1929). Process and Reality: An Essay in Cosmology. NY: Macmillan Company.

Wierzbicki, A. P., \& Nakamori, Y. (2007). Creative Environments. Issues of Creative Support for the Knowledge Civilization Age. Studies in Computational Intelligence (Vol. 59). Springer.

Wynn, D. C., \& Clarkson, P. J. (2017). Process Models in Design and Development. Research in Engineering Design, 29(2), 161-202.

Publisher's Note Springer Nature remains neutral with regard to jurisdictional claims in published maps and institutional affiliations. 